\title{
A 9-Year-Old Patient with Recurrent Fever, Urticarial Rash and Demyelinating Brain Lesions: NLRP3-Autoinflammatory Disease in Ecuador
}

\section{Cristina N Herrera \\ Hospital Roberto Gilbert Elizalde, Servicio de Reumatología, Guayaquil, Ecuador}

\begin{abstract}
Autoinflammatory diseases (AIDs) are a relatively new family disorders defined approximately 20 years ago. AIDs are caused by defect(s) or dysregulation of the innate immune system, characterized by recurrent or continuous inflammation and lack of a primary pathogenic role for the adaptive immune system. One AID, NLRP3-associated autoinflammatory disease (NLRP3-AID), involves a clinical presentation since the neonatal period or childhood, with multiple inflammatory recurrent symptoms that appear throughout the patient's life. We present the first case of NLRP3-AID in Ecuador. The patient presented recurrent fever since 6 months of age associated with urticarial rash, arthralgias, and abdominal pain; recently, he had a seizure at 7 years of age. Brain MRI revealed demyelinating lesions, and genetic testing uncovered a de novo mutation in the NLRP3 gene. The patient had a good clinical response to treatment with canakinumab.
\end{abstract}

Keywords: demyelinating diseases, NLRP3-autoinflammatory disease, Ecuador

\section{Background}

NLRP3-AID is an uncommon disease with an incidence of 1 in 1,000,000 people. Clinical manifestations appear during pediatric ages (mostly before the 2 nd year of life). Biological markers, such as C-reactive protein, are increased, revealing an inflammatory process. Genetical testing helps to diagnose this entity. NLRP3-AID encompasses 3 clinical entities with different levels of phenotypic severity (mild, moderate and severe).

\section{Case Report}

We report a case of a seven-year-old child who presented recurrent episodes of fever since 6 months of age but that were not periodic (he was febrile for 7 or 21 days and afebrile for 3 or 7 days). In addition, he experienced recurrent episodes of painless red eyes, urticarial rash on the legs and chest (Figure 1A), myalgias and arthralgias, but without arthritis or joint limitation.

Hematologists who were consulted excluded myeloproliferative disease; adult rheumatologists had diagnosed him with juvenile idiopathic arthritis and treated him with NSAIDs, methotrexate, and corticosteroids (pulsed and oral). Subsequently, the patient received a single dose of tocilizumab, which was suspended due to an infusion reaction. He also received rituximab. No medication was successful. 

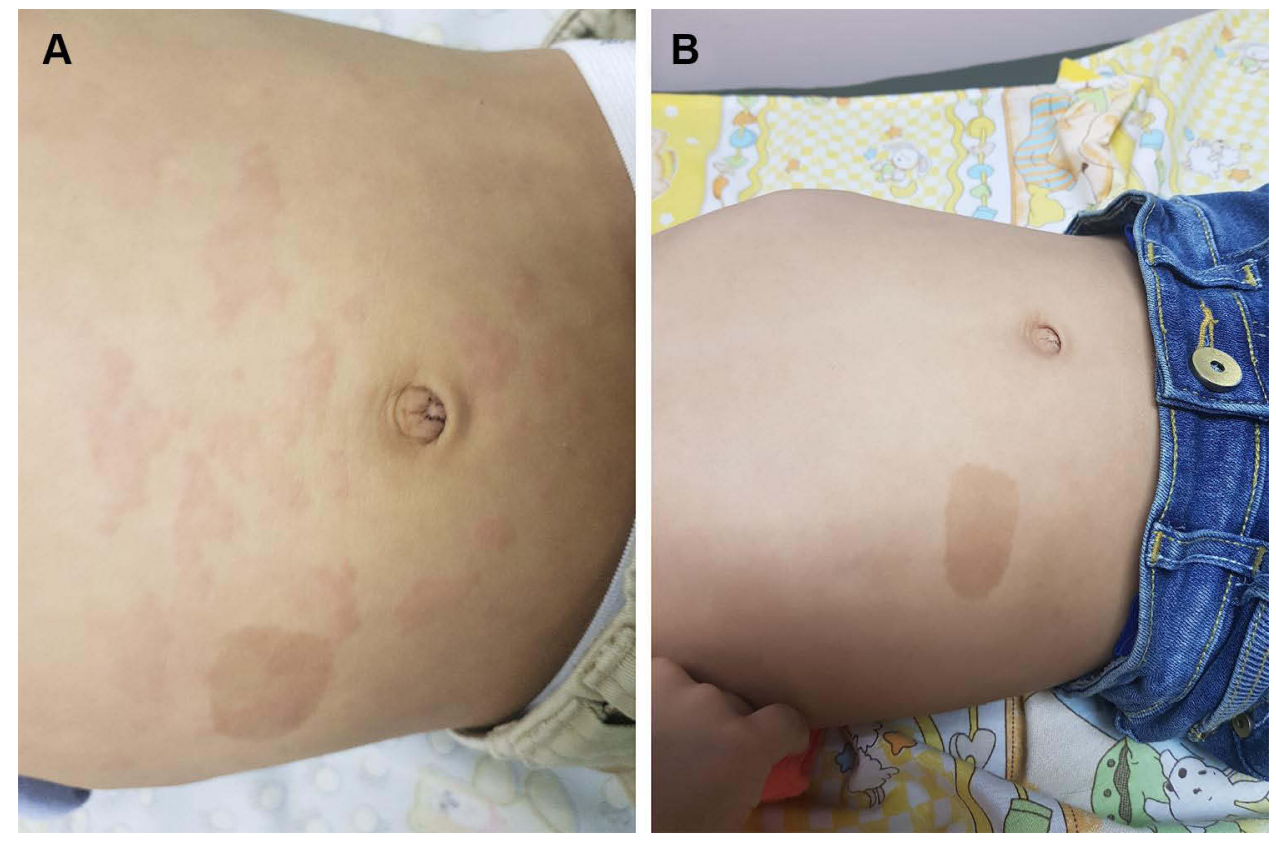

Figure I (A) Urticarial rash on the chest before treatment. (B) After treatment, the rash has disappeared.

Finally, he visited our hospital at the age of 6 years. He was febrile, and physical examination revealed red eye, urticarial rash, frontal bossing, hypertrophic tonsils, watch crystal nails and failure to thrive. Laboratory tests showed marked leukocytosis (between 17,000 and 25,000 per $\mathrm{mm}^{3}$ ), anemia $(\mathrm{Hb} 8.7 \mathrm{mg} / \mathrm{dL})$, a high C-protein reactive (CPR) and erythrocyte sedimentation rate (ESR), and no proteinuria or hematuria (urine); he was negative for anti-Smith, anti-DNA, and anti-RNP antibodies. PPD skin test was negative, and his thorax radiograph was normal. Serologies for Epstein Barr virus, Toxoplasmosis, Herpes virus I and II, Cytomegalovirus, HIV, Hepatitis B and $\mathrm{C}$ were negative. He was evaluated by ophthalmology, and corneal subepithelial infiltrates were found in both eyes as well as nummular keratitis.

Due to clinical data, the duration of symptoms, and having ruled out other infectious and myeloproliferative diseases, a genetic test was performed to the parents and the patient with collaboration of the Genetics Laboratory of the Giannina Gaslini Hospital (Genoa, Italy). The amplification products of exons 3-5 and exon 3 of the TNFRSF1A and NLRP3 genes respectively, were sequenced with the following results: TNFRSF1A gene, no mutation. NLRP3 gene (NM_001243133.1): L571FG $>C$ variant, leading to the missense $p$. Leu571Phe, was detected. This variant is likely pathogenic and was not present in the parents. A whole-genome sequencing was not performed. Therefore, a diagnosis of
NLRP3-AID was made when the patient was 7 years old. However, due to a lack of anti-IL1 in Ecuador, he did not start treatment immediately.

When he was 7 and a half years old, he experienced two generalized tonic-clonic seizures with loss of consciousness. He did not have other neurologic nor psychiatric symptoms, he was not taking any medication at that time; he presented fever and urticarial rash, though his arterial pressure was normal. Lumbar puncture analysis was normal. A new ophthalmologic evaluation reported scattered punctate subepithelial leukoma, and the borders of the optic nerve in the fundus were blurred, suggesting neuritis. MRI of the brain revealed a large hyperintense lesion in the right occipital lobe and other small lesions in the left parietal and temporal lobes; the large one captured contrast. Such lesions suggest demyelinating lesions (Figure 2A-C), he received diphenylhydantoin and pulses of corticosteroids. He had no more seizures.

At 8 years old, owing to a foreign donation, treatment with canakinumab (anti-IL1) was started at $2 \mathrm{mg} / \mathrm{kg}$ every 8 weeks; one hour after the application, the rash and red eye subsided. He did not present fever again. Prior to treatment, CRP was between $82.4 \mathrm{mg} / \mathrm{dL}$ and $267 \mathrm{mg} / \mathrm{dL}$; subsequently, CRP decreased to $5.2 \mathrm{mg} / \mathrm{dL}$, and $\mathrm{Hb}$ normalized $(11.5 \mathrm{mg} / \mathrm{dL})$. Three months later, a brain MRI revealed improvement of the brain lesions (Figure 2D and E). His electroencephalogram was normal; diphenylhydantoin was discontinued, after it had been used for 1 year. 

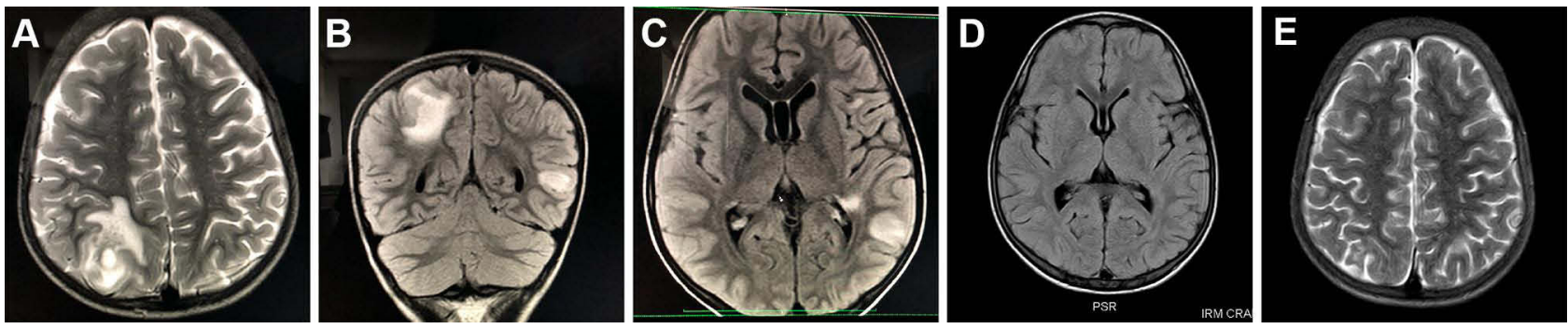

Figure 2 (A-C) brain MRI revealed a large hyperintense lesion in the right occipital lobe and other small lesions in the left parietal and temporal lobes; the large one was contrast- enhancing. (D and $\mathbf{E})$ Three months later, a brain MRI revealed improvement of the brain lesions.

Otolaryngology recommended tonal audiometry and impedance audiometry, but the parents did not agree.

The patient has used canakinumab for 2 years, without adverse events; clinically, he has not experienced fever, rash (Figure 1B), abdominal pain, red eye, or seizure. He is still followed up by our Neurology Department. The patient had failed to thrive, and his weight pretreatment was $18 \mathrm{~kg}$ and his height $111.7 \mathrm{~cm}$; after 2 years of treatment, he weighed $31 \mathrm{~kg}$ and was $127.5 \mathrm{~cm}$ tall. Physical examination still showed watch crystal nails and hypertrophic tonsils, but without episodes of sleep apnea. He had no developmental delay.

The Roberto Gilbert Elizalde Hospital ethics committee does not require ethical approval for case reports. Written informed mother's patient consent to publish the case, photos and brain MRI was obtained.

\section{Discussion}

Autoinflammatory disorders are a group of medical disorders derived from defects or dysregulation of the innate immune system and are characterized by recurrent or continuous inflammation (elevated acute-phase reactants) and the lack of a primary pathogenic role for the adaptive immune system (autoreactive T-cells or autoantibody production). NLRP3- AID was previously called cryopyrinassociated periodic syndrome (CAPS). The last consensus changes the name to NLRP3-AID, which does not contain the word "periodic" (when the frequency of the attacks is relatively regular) because the attacks are not periodic but recurrent (without a regular pattern). ${ }^{1-4}$

The diagnosis of AIDs is based on the interpretation of clinical phenotype and molecular genetic analysis. Nevertheless, many patients develop phenotypes that do not fit any clinically and/or genetically defined disorders; hence, it is important to acquire a full genomic profile through whole-exome and/or genome sequencing. ${ }^{5}$ The New Eurofever/PRINTO Classification criteria for autoinflammatory recurrent fevers were established in 2019. For NLRP3-AID classification, the presence of a confirmatory NLRP3 genotype plus at least one criterion is required (urticarial rash, red eye (conjunctivitis, episcleritis, uveitis), neurosensorial hearing loss) or nonconfirmatory NLRP3 genotype plus at least 2 criteria (urticarial rash, red eye, and neurosensorial hearing loss). ${ }^{6}$ These criteria should be applied if other causes of fever (infections, immunodeficiency, neoplasms, other rheumatic diseases including juvenile idiopathic arthritis) have been excluded. ${ }^{1}$ In our patient, myeloproliferative disease and infections were ruled out. Infectious diseases such as dengue and chikungunya are common in Ecuador, but in these cases, fever does not last more than 2 weeks. Tuberculosis is endemic, so a chest $\mathrm{x}$-ray and PPD skin test were performed and were normal. Other infections such as toxoplasmosis, hepatitis, cytomegalovirus, Epstein-Barr and HIV were also excluded. The patient underwent his first evaluation in our hospital at 6 years of age, and he did not present limitations or palpable synovitis over time. Clinical suspicion motivated the molecular test.

Mutations in NLRP3 [the NOD (nucleotide-binding oligomerization domain)-like receptor family gene, pyrin domain 3] and its product NALP3 (Nacht leucine-rich repeat domain and pyrin domain containing protein 3 , or cryopyrin) are associated with NLRP3-AID. The NLRP3 gene (also referred to as C1AS1, PYPAF1, NALP3) encodes a protein called cryopyrin 2 ; in this case, the c. $1713 \mathrm{G}>\mathrm{C}$ mutation was present. The disease autoinflammatory due to excess production of interleukin (IL)- $1 \beta$, which is an innate immune cytokine and potent endogenous pyrogen that causes a cytokine amplification cycle. ${ }^{2}$

Clinical entities encompassed by NLRP3 show increasing degrees of severity: mild (previously called familial cold-induced urticaria), moderate (previously Muckle Wells syndrome) and severe (previously CINCA [chronic 
infantile neurologic, cutaneous and articular]/NOMID (neonatal onset multisystemic inflammatory disease). ${ }^{3,4,7}$

Severe forms are characterized by arthropathy, urticaria, central nervous system (CNS) involvement, perceptual deafness, and visual impairment; renal amyloidosis may also occur. The age of presentation is in the neonatal period. Moderate forms present urticaria, fever, arthralgia, headache, conjunctivitis, sensorineural deafness, and renal amyloidosis. ${ }^{3,7}$ The manifestations of our patient were compatible with clinical and imaging data: recurrent (not periodic) fever, urticarial rash, CNS alteration, arthralgia, and conjunctivitis. The NLRP3 genotype supported the diagnosis.

The neurological manifestations of NLRP3-AID found in our patient (seizures and sclerosis-like lesions) have been reported before. Lequerré et al described 4 generations (11 members) of a family with NLRP3-AID. Two members developed multiple sclerosis-like neurological signs concomitant with urticaria, arthritis and episcleritis, and none had a low IQ or chronic meningitis. ${ }^{8}$ CompeyrotLacassagne also reported a case of a 45-year-old woman with Muckle Wells syndrome. Her clinical features were chronic headaches, chronic tinnitus, conjunctivitis, urticarial rash, arthralgia, nondestructive arthritis, myalgia and chronic fatigue. Brain MRI showed demyelination in the corpus callosum and temporal lobe. ${ }^{9}$

Kilic et al studied 12 patients with NLRP3-AID, of whom half had neurological manifestations: headache ( $\mathrm{n}=$ $4 / 12)$, seizures $(n=3 / 12)$, papilledema $(n=3 / 12)$, intellectual disability $(n=2 / 12)$, aseptic meningitis $(n=2 / 12)$, hearing loss $(n=2 / 12)$, and optic atrophy $(n=1 / 12)$. MRI revealed alterations in only 2 cases. With the use of antiIL1, Kilic reported complete resolution of symptoms and prevention of progression of the neurological signs when medication was initiated early. ${ }^{10}$

Brain MRI showing an acute demyelinating encephalomyelitis-like syndrome has been described in cerebral autoinflammatory diseases with good response to anti-IL -1. Jang et al reported the case of a 51-year-old patient in whom they performed a brain biopsy finding massive CD68/CD163 positive microglial proliferation. Microglia, as well as other cells of innate immunity, produce IL1; based on which microglia were targeted. ${ }^{11}$

Kenney-Jung et al described a case with favorable response to anti-IL1 (anakinra) in a 32-month-old girl with super refractory status epilepticus and febrile infection-associated epilepsy syndrome (FIRES). She had intrathecal overproduction of IL6 and IL8 cytokines, with the use of anakinra she achieved normalization of IL6, IL8 in cerebrospinal fluid suggesting inhibition of the iL1-1R1 by anakinra blocked a down-stream signaling cascade responsible for their central nervous system production. These cases clarify how inflammation occurs in AIDs and the cells involved. ${ }^{12}$

Approved treatments for NLRP3-AID are anakinra (recombinant homolog of IL1 receptor antagonist) and canakinumab (humanized anti-IL1B monoclonal antibody). Both are effective in controlling the disease. Canakinumab has the advantage of being administered every 8 weeks, whereas anakinra is administered daily; in addition, canakinumab produces fewer local reactions in the area of injection. ${ }^{13,14}$ Neither anakinra nor canakinumab are available in Ecuador. Thanks to a Managed Access Program, canakinumab was donated for our patient as compassionate use. He was able to start treatment, followed by clinical remission and normal brain MRI results.

\section{Conclusion}

Although NLRP3-AID is a well-defined syndrome, it is not very well known in undeveloped countries, which leads to late diagnosis and treatment. Neurological manifestations in NLRP3-AID are possible even in moderate forms.

\section{Acknowledgments}

We would like to thank the Molecular Biology Department of Roberto Gilbert Hospital for their collaboration with DNA extraction and sample storage and the Genetics and Rheumatology Department of Giannina Gaslini Hospital (Genoa, Italy) for the genetic analysis.

\section{Disclosure}

The author reports grants from Roche outside the submitted work and reports no other conflicts of interest in this work.

\section{References}

1. Federici S, Sormani MP, Ozen S, et al. Evidence-based provisional clinical classification criteria for autoinflammatory periodic fevers. Ann Rheum Dis. 2015;74(5):799-805. doi:10.1136/annrheumdis2014-206580

2. Haverkamp MH, Van de Vosse E, Goldbach-Mansky R, Holland SM. Impaired cytokine responses in patients with cryopyrin-associated periodic syndrome (CAPS). Clin Exp Immunol. 2014;177 (3):720-731. doi:10.1111/cei.12361

3. Sarrabay G, Grandemange S, Touitou I. Diagnosis of cryopyrin-associated periodic syndrome: challenges, recommendations and emerging concepts. Expert Rev Clin Immunol. 2015;11 (7):827-835. 
4. Ben-Chetrit E, Gattorno M, Gul A, et al. Consensus proposal for taxonomy and definition of the inflammatory diseases (AIDs) a Delphi study. Ann Rheum Dis. 2018;77(11):1558-1565. doi:10.1136/annrheumdis-2017-212515

5. Tucker LB, Lamot L, Niemietz I, et al. Complexity in unclassified auto-inflammatory disease: a case report illustrating the potential for disease arising from the allelic burden of multiple variants. Pediatr Rheumatol Online J. 2019;17(1):70. doi:10.1186/s12969019-0374-x

6. Gattorno M, Hofer M, Federici S, et al; Eurofever Registry and the Paediatric Rheumatology International Trials Organisation (PRINTO). Classification criteria for autoinflammatory recurrent fevers. Ann Rheum Dis. 2019;78(8):1025-1032. doi:10.1136/annrheumdis-2019-215048

7. Levy R, Gérard L, Kuemmerle-Deschner J, et al. Phenotypic and genotypic characteristics of cryopyrin-associated periodic syndrome: a series of 136 patients from the eurofever registry. Ann Rheum Dis. 2014:1-7. doi:10.1136/annrheumdis-2013-203953

8. Lequerré T, Vittecoq O, Saugier-Veber P, et al. A cryopyrin-associated periodic syndrome with joint destruction. Rheumatology. 2007;46:709-7 14. doi:10.1093/rheumatology/kel399
9. Compeyrot-Lacassagne S, Tran TA, Guillaume-Czitrom S, Marie I, KonéPaut I. Brain multiple sclerosis-like lesions in a patient with muckle-wells syndrome. Rheumatology. 2009;48(12):1618-1619. doi:10.1093/rheumatology/kep321

10. Kilic H, Sahin S, Duman C, et al. Spectrum of the neurologic manifestations in childhood-onset cryopyrin-associated periodic syndrome. Eur J Paediatr Neurol. 2019;23(3):466-472. doi:10.1016/j.ejpn.2019.03.006

11. Jang Y, Woo K, Lee ST, Park SH, Chun K, Kun lee S. Cerebral autoinflammatory disease treated with anakinra. Ann Clin Transl Neurol. 2018;5(11):1428-1433. doi:10.1002/acn3.656

12. Kenney-Jung D, Kahoud R, Vezzani A, et al. Super-refractory status epilepticus and febrile infection-related epilepsy syndrome treated with anakinra. Ann Neurol. 2016;80(6):939-945. doi:10.1002/ana.24806

13. Yokota S, Nishikomori R, Takada H, et al. Guidance on the use of canakinumab in patients with cryopyrin-associated periodic syndrome in Japan. Mod Rheumatol. 2013;23:425-429. doi:10.3109/s10165-0120769-8

14. Treviño SJ, Polo ER. Tratamiento de los síndromes periódicos asociados a la criopirina (CAPS). Med Clin. 2011;136(Supl1):29-33. doi:10.1016/S0025-7753(11)70006-9
Open Access Rheumatology: Research and Reviews

\section{Publish your work in this journal}

Open Access Rheumatology Research and Reviews is an international, peer-reviewed, open access journal publishing original research, reports, editorials, reviews and commentaries on all aspects of clinical and experimental rheumatology in the clinic and laboratory including the following topics: Pathology, pathophysiology of rheumatological diseases; Investigation, treatment and management

\section{Dovepress}

of rheumatological diseases; Clinical trials and novel pharmacological approaches for the treatment of rheumatological disorders. The manuscript management system is completely online and includes a very quick and fair peer-review system, which is all easy to use. Visit http://www.dovepress.com/testimonials.php to read real quotes from published authors. 